\title{
Segurança do paciente: representações sociais na visão dos profissionais da atenção primária à saúde
}

\section{Patient safety: social representations from the perspective of primary health care professionals}

\section{Seguridad del paciente: representaciones sociales de la percepción de los profesionales de la atención primaria de salud}

\author{
Samira Valentim Gama Lira de Alencar iD \\ Universidade de Fortaleza - Fortaleza (CE) - Brasil \\ Emanuel Alves do Nascimento iD \\ Cooperativa Univida - Maranguape (CE) - Brasil \\ Charlyanne Diógenes Brito (iD \\ Hospital e Maternidade Adolfo Bezerra de Menezes - Jaguaretama (CE) - Brasil \\ Daiany Dântara de Sousa Barbosa iD \\ Davita Serviços de Nefrologia São Gerardo - Fortaleza (CE) - Brasil \\ Lívia de Andrade Marques \\ Universidade de Fortaleza - Fortaleza (CE) - Brasil \\ Geisy Lanne Muniz Luna iD \\ Escola de Saúde Pública do Ceará - Fortaleza (CE) - Brasil \\ Débora Rodrigues Guerra Probo iD \\ Universidade de Fortaleza - Fortaleza (CE) - Brasil \\ Ricardo Augusto da Silva Probo (iD \\ Learning, Development \& Sintony - LDS Treinamentos - Fortaleza (CE) - Brasi
}

\section{RESUMO}

Objetivo: Apreender as representações sociais da segurança do paciente sob a ótica dos profissionais de saúde inseridos no contexto da Atenção Primaria à Saúde. Métodos: Trata-se de um estudo com abordagem qualitativa, usando o referencial teórico-metodológico da teoria das representações sociais, realizado no município de Fortaleza, Ceará, Brasil. Fizeram parte do estudo 18 participantes sociais da Estratégia Saúde da Família. A coleta dos dados ocorreu de setembro a outubro de 2019, por meio da aplicação de uma entrevista semiestruturada, sendo analisados por meio do método de análise de conteúdo temático, do qual emergiram as seguintes categorias: "Representações sociais da segurança do paciente na Estratégia Saúde da Família"; "Representações sociais do risco e estratégia preventiva na segurança do paciente na Estratégia Saúde da Família"; "Fragilidades da segurança do paciente na Estratégia Saúde da Família". Resultados: As representações sociais apreendidas por meio das falas caracterizam a segurança do paciente como espaço social de interação entre profissionais e pacientes. Os profissionais, ao exercerem sua prática, utilizam recursos para melhorar o atendimento, mas ressaltam a existência de riscos, sendo importante desenvolver estratégias preventivas como técnicas adequadas, desinfecção e esterilização. Além disso, destacam a presença de fragilidades na rotina de trabalho que interferem na garantia da segurança do paciente, como estrutura física e falta de materiais. Conclusão: Os profissionais de saúde demonstram, por meio da ancoragem e da objetivação, devem aplicar os conceitos da segurança do paciente nos cenários sociais, mas necessitam que os gestores disponibilizem insumos e estrutura que favoreçam essa assistência.

Descritores: Atenção Primária à Saúde; Segurança do Paciente; Estratégia Saúde da Família.

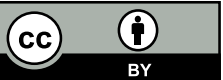




\section{ABSTRACT}

Objective: To apprehend the social representations of patient safety from the perspective of health professionals inserted in the context of Primary Health Care. Methods: This is a qualitative study that used the theoretical and methodological framework of the theory of social representations carried out in the city of Fortaleza, Ceará, Brazil. A total of 18 social participants of the Family Health Strategy were part of the study. Data were collected from September to October 2019 through semi-structured interviews and were analyzed using the thematic content analysis method, from which the following categories emerged: "Social representations of patient safety in the Family Health Strategy"; "Social representations of risk and preventive strategy for patient safety in the Family Health Strategy"; "Weaknesses in patient safety in the Family Health Strategy". Results: The social representations apprehended from the Family Health Strategy professionals' accounts characterize patient safety as a social space for professionals and patients to interact. Professionals, in their practice, use resources to improve care, but emphasize the existence of risks, and it is important to develop preventive strategies such as appropriate techniques, disinfection and sterilization. In addition, they highlight the presence of weaknesses in the work routine that interfere with patient safety, such as physical structure and lack of materials. Conclusion: Health professionals demonstrate, through anchoring and objectification, that they must apply the concepts of patient safety in social settings, but they need managers to provide input and structure that favor the provision of care.

Descriptors: Primary Health Care; Patient Safety; Family Health Strategy.

\section{RESUMEN}

Objetivo: Aprehender las representaciones sociales de la seguridad del paciente bajo la visión de los profesionales sanitarios inseridos en el contexto de la Atención Primaria de Salud. Métodos: Se trata de un estudio de abordaje cualitativo con el referencial teórico-metodológico de la teoría de las representaciones sociales realizado en el municipio de Fortaleza, Ceará, Brasil. Participaron del estudio 18 participantes sociales de la Estrategia de Salud de la Familia. La recogida de datos se dio entre septiembre y octubre de 2019 a través de una entrevista semiestructurada analizada por el método de análisis de contenido temático del cual emergieron las siguientes categorias: "Representaciones sociales de la seguridad del paciente de la Estrategia Salud de la Familia", "Representaciones sociales del riesgo y la estrategia de prevención para la seguridad del paciente de la Estrategia Salud de la Familia"; "Fragilidades de la seguridad del paciente de la Estrategia Salud de la Familia". Resultados: Las representaciones sociales aprehendidas de las hablas de los participantes caracterizan la seguridad del paciente como el espacio social de interacción entre los profesionales y pacientes. En sus prácticas los profesionales utilizan recursos para mejorar la atención pero también destacan la existencia de riesgos y, por ello, es importante el desarrollo de estrategias de prevención como las técnicas adecuadas, la desinfección y la esterilización. Además de eso, los profesionales destacan la presencia de fragilidades en su rutina de trabajo como la estructura física y la falta de materiales que interfieren para la garantía de la seguridad del paciente. Conclusión: Los profesionales sanitarios han demostrado a través del ancoraje y de la objetivación, que deben aplicar los conceptos de la seguridad del paciente en los escenarios sociales pero necesitan la disponibilidad de insumos $y$ estructura de parte de los gestores que favorezcan esa asistencia.

Descriptores: Atención Primaria de Salud; Seguridad del Paciente; Estrategia de Salud Familiar.

\section{INTRODUÇÃO}

A partir da publicação da Política Nacional de Segurança do Paciente no Brasil, em 2013, os diversos serviços precisaram se adaptar para o cumprimento da legislação sanitária vigente, que decorreu de uma Aliança Mundial de Segurança do Paciente, cumprindo recomendações da Organização Mundial de Saúde (OMS) ${ }^{(1,2)}$.

AAtenção Primária à Saúde (APS) oportunizou, no Brasil, a ampliação da cobertura populacional pelas equipes de saúde da família, proporcionando acesso à atenção integral, e se configura como um serviço que funciona como porta de entrada e organizadora do Sistema Único de Saúde SUS) ${ }^{(3)}$.

Conforme a Política Nacional de Promoção da Saúde, os profissionais de saúde devem promover e melhorar as condições de saúde e modos de viver em sua assistência, ampliando a potencialidade da saúde individual e coletiva, garantindo a segurança do paciente diante da redução das vulnerabilidades e dos riscos à saúde decorrentes dos determinantes sociais, econômicos, culturais, psicológicos e comportamentais ${ }^{(4)}$.

A prestação de cuidados primários seguros é prioritária, pois todos os dias milhões de pessoas utilizam os serviços de APS em todo o mundo. Por isso, garantir a segurança do paciente nesse serviço é essencial quando se busca alcançar a saúde universal, cobertura e sustentabilidade dos cuidados de saúde. $O$ atendimento primário seguro melhora a saúde e o bem-estar de indivíduos, comunidades e sociedades ${ }^{(5)}$.

$\mathrm{A} \mathrm{OMS}^{(6)}$ ressalta que as falhas na atenção primária contribuem para o ônus da atenção globalmente insegura e estima que $80 \%$ dos danos das unidades básicas de saúde possam ser evitados. Ressalta ainda que os cuidados 
inseguros e de baixa qualidade é uma das razões pelas quais os indivíduos normalmente ignoram os cuidados primários, optando pelos cuidados secundários ${ }^{(6)}$.

A Aliança Mundial para a Segurança do Paciente, criada pela OMS em 2004(7), possui como objetivo adotar medidas de melhoria no atendimento ao paciente e aumentar a qualidade dos serviços de saúde, e dentre os países politicamente comprometidos com os propósitos da OMS encontra-se o Brasil. Logo depois, no âmbito da Agência Nacional de Vigilância Sanitária, a Resolução da Diretoria Colegiada (RDC) n. ${ }^{\circ} 36$, institui ações para a segurança do paciente em serviços de saúde e dá outras providências, contribuindo para o núcleo de segurança do paciente ao estabelecer estratégias e ações de gestão de risco, conforme as atividades desenvolvidas pelo serviço de saúde ${ }^{(1)}$.

Comumente, a questão da segurança do paciente é mais abordada e discutida no ambiente hospitalar, muito provavelmente, devido aos maiores riscos em função de cuidados mais complexos, com maior aporte tecnológico e custos elevados ${ }^{(8)}$. Contudo os problemas com a segurança do paciente não ocorrem apenas em ambiente hospitalar, podendo ocorrer em outros espaços, como serviços especializados, atenção primária, ambiente domiciliar e nos atendimentos pré-hospitalares. Por isso, a conduta na garantia da segurança do paciente deve ser mantida independentemente do local no qual é prestado o atendimento ${ }^{(9-11)}$.

A avaliação da cultura de segurança na APS é multifatorial e vai além dos elementos relacionados à estrutura, processo e resultados do trabalho em saúde. Ressalta-se a necessidade de fortalecer as discussões de segurança do paciente na APS para promover a conscientização dos profissionais sobre seu compromisso ético e comunicação efetiva, visando fortalecer a assistência ${ }^{(12)}$.

Os profissionais de saúde da APS podem ter altos padrões pessoais de cuidado sem ter consciência dos riscos aos quais os pacientes estão expostos no sistema de saúde como um todo, e o conceito de segurança do paciente talvez não possua uma boa repercussão entre os profissionais da APS. Assim, as estratégias que visam melhorar a segurança do paciente nesse nível de atenção devem enfatizar o envolvimento do paciente, a coordenação e cooperação da equipe, e uma mentalidade de previsão, monitoramento e cuidado com o paciente para além da sua internação ou consulta(13). Entretanto, utiliza-se como estratégia para o desenvolvimento seguro na atenção básica, como a ética profissional e o acolhimento, como fatores que colaboram para a segurança do paciente, visando a preocupação com os procedimentos técnicos, como a lavagem das mãos, a não contaminação e o uso de equipamentos de proteção individual ${ }^{(14)}$.

Dessa forma, é possível perceber que APS vem desempenhando um papel cada vez mais complexo no sistema, acarretando maior risco de incidentes ${ }^{(7)}$. Por isso, é fundamental que os profissionais de saúde ofereçam um cuidado seguro, livre de qualquer dano, sendo capaz de identificar as possíveis falhas e buscar soluções que visem ao cuidado efetivo e com segurança.

Diante disso, este estudo optou por se basear na teoria das representações sociais, que reflete uma forma de saber organizado que promove a constituição de uma realidade comum a determinado grupo social. Assim, portanto, é uma maneira de compreender nuances e processos de conhecimentos, partilhados por profissionais de saúde, acerca da segurança do paciente em seu cotidiano de trabalho ${ }^{(15)}$.

As pesquisas acadêmicas sobre segurança do paciente são menos frequentes na APS, sendo exploradas principalmente na atenção terciária, no ambiente hospitalar ${ }^{(8)}$. Em determinados países, como os Estados Unidos, Austrália e Portugal, a segurança do paciente vem sendo avaliada e explorada. No Brasil, a temática ganhou destaque em 2013, a partir da implantação do Programa Nacional de Segurança do Paciente, e deve-se ponderar que ainda há pouca dedicação em estudos brasileiros nesse cenário(16). Esta é a relevância e a importância do presente trabalho: alertar os profissionais, os gestores e a população sobre a temática da segurança do paciente na atenção primária. Nessa direção, foi traçada a seguinte questão de pesquisa: Como se apresentam para os profissionais da Estratégia Saúde da Família, inseridos no contexto social da APS, as ações na garantia da segurança do paciente?

Portanto, esta pesquisa teve como objetivo apreender as representações sociais da segurança do paciente sob a ótica dos profissionais de saúde inseridos no contexto da Atenção Primaria à Saúde.

\section{MÉTODOS}

Trata-se de uma pesquisa qualitativa ${ }^{(17)}$ que teve como referencial teórico-metodológico a teoria das representações sociais ${ }^{(18,19)}$, que se baseia em dois princípios para tornar familiar elementos que são desconhecidos: a ancoragem, que relaciona o novo a um conhecimento prévio, e a objetivação, que atribui significado a uma ideia, redefinindo a relação sujeito-objeto, por meio de uma esquema do âmbito conceitual e intelectual para o material. Por meio das 
representações sociais é possível entender os fenômenos que nos permitem identificar a relação entre as pessoas e o mundo que vivem ${ }^{(20)}$. No caso do presente estudo, essa construção social se faz por meio da formação profissional e do cotidiano laboral em serviços da Estratégia Saúde da Família (ESF).

A pesquisa foi realizada no município de Fortaleza, Ceará, Brasil, no período de setembro a outubro do ano de 2019. No momento da pesquisa, o município possuía 113 Unidades de Atenção Primária à Saúde (UAPS), e se realizou um sorteio para selecionar o serviço( ${ }^{(21)}$. Os participantes desta pesquisa foram profissionais da área da saúde pertencentes à AP, sendo incluídos somente profissionais que faziam parte da equipe de ESF, sendo excluídos: profissionais de saúde com cargos administrativos, profissionais do Núcleo de Apoio à Saúde da Família (NASF) e profissionais ESF, na categoria de agente comunitário de saúde (ACS), assim como os que estavam afastados, de férias ou de licença. Conforme os critérios de inclusão e exclusão, totalizava-se 35 profissionais de saúde. Destes, 12 não aceitaram participar da pesquisa, devido a não possuir disponibilidade de tempo durante o trabalho e por não pretender contribuir com a pesquisa; três estavam de licença à saúde e dois estavam de férias. Assim, ao final, o estudo foi composto por 18 profissionais, os quais foram denominados por "Profissional", seguido por um número, em ordem sucessiva, para garantir o anonimato.

Utilizou-se na coleta de dados uma entrevista semiestruturada(17), em que se propôs investigar as seguintes questões: características sociodemográficas (profissão, sexo, idade, naturalidade, renda e religião) e a seguinte questão norteadora: como se apresenta para os profissionais da Estratégia Saúde da Família, inseridos no contexto social da APS, ações na garantia da segurança do paciente?

A pesquisa ocorreu nas dependências das UAPS, onde inicialmente, os pesquisadores contataram a coordenação para explicar o estudo e organizar um cronograma para a coleta de dados a partir das escalas dos profissionais de saúde no serviço. Assim, os profissionais de saúde receberam a informação da realização da pesquisa, ficando cientes de sua colaboração. Então, os pesquisadores, durantes várias semanas, diante do cronograma préestabelecido, contataram os participantes, os quais aceitaram ou não participar da pesquisa. A entrevista foi agendada fora do horário de expediente, sendo realizada individualmente, com pesquisador e entrevistado, em clima amistoso em consultório, para preservar a individualidade e singularidade do participante, com duração em média de 15 minutos. As falas dos entrevistados foram gravadas e transcritas, mediante a autorização e assinatura do Termo de Consentimento Livre e Esclarecido (TCLE).

Os dados foram analisados utilizando-se o método de análise de conteúdo temático, que abrange as seguintes etapas: pré-análise, que consiste na fase de organização dos dados, tendo por objetivo sistematizar as ideias; exploração do material, na qual se inicia o processo de codificação, classificação e categorização das informações; e tratamento dos resultados, em que se realiza a inferência e interpretação dos resultados, procurando torna-los válidos e significativos ${ }^{(22)}$.

As categorias empíricas construídas ao final da operação de análise consistem em três categorias temáticas: "Representações sociais da segurança do paciente na Estratégia Saúde da Família"; "Representações sociais do risco e estratégia preventiva na segurança do paciente na Estratégia Saúde da Família"; "Fragilidades da segurança do paciente na Estratégia Saúde da Família".

O estudo beseia-se nos princípios legais e éticos adotados nas investigações que envolvem seres humanos, como preconiza a Resolução n. ${ }^{\circ} 466 / 2012$, do Conselho Nacional de Saúde ${ }^{(23)}$, tendo sido aprovada pelo Comitê de Ética da Universidade de Fortaleza com o Parecer n. ${ }^{\circ}$ 3.645.205. Cada participante foi devidamente orientado quanto aos objetivos da pesquisa, tendo lido e assinado o TCLE.

\section{RESULTADOS}

\section{Dados sociodemográficos dos entrevistados}

O sexo predominante foi o feminino (17 participantes), sendo apenas um do masculino. Participaram profissionais de ensino superior e médio, sendo eles: enfermeiros, cirurgiões-dentistas, médicos, técnico de enfermagem, auxiliar em saúde bucal. A renda dos profissionais variou entre 1 a 15 salários mínimos, sendo mais citado a renda entre 1 e 2 salários mínimos. Foram mencionadas a religião católica, cristã e evangélica, sendo a mais citada a religião católica. Acerca do tempo de trabalho nas UAPS, variou em torno de 5 a 20 anos, e o tempo de formados variou de 8 a 29 anos.

\section{Representações sociais da segurança do paciente na Estratégia Saúde da Família}

Nessa categoria, discutem-se aspectos sobre o conhecimento de segurança do paciente, destacando-se ações que os profissionais realizam no seu dia a dia, comprovando a adoção dessa prática. 
Dessa forma, os profissionais relataram, em relação aos seus conhecimentos prévios sobre a segurança do paciente, nos quais se referiram aos documentos existentes ${ }^{(1-3)}$, que visam diminuir ou evitar que o paciente sofra algum dano, tendo suas respostas evidenciadas nas seguintes falas:

"Realizar todas as atividades que Ihe são peculiares, as normas, rotinas e protocolos estabelecidos, assim como os profissionais também devem obedecer e seguir as rotinas ao manter os cuidados ao mesmo." (Profissional 4)

"[...] conjunto de medidas que visam diminuir ou evitar completamente a exposição do paciente a riscos; riscos de integridade física, riscos de acidentes, riscos de expor a infecções." (Profissional 10)

"Proporcionar para o paciente condições que, no atendimento, ele não seja exposto a nenhum tipo de injúria, que não tenha nenhuma intercorrência durante ao atendimento." (Profissional 14)

Além disso, eles comentaram sobre a rotina em seu contexto de trabalho, dando ênfase à segurança do paciente e percebendo-a em pequenas ações vivenciadas diariamente, utilizando todos os recursos para um melhor atendimento:

"A partir do momento em que a gente acolhe o paciente [...]" (profissional 3)

"No dia a dia, [...] com todos os funcionários que procuram usar todos os equipamentos para manter a segurança do paciente. [...] A gente procura utilizar todos os recursos para manter a segurança do paciente sim." (Profissional 9)

"[...] a segurança em relação ao paciente é uma coisa que é bem trabalhada, tanto durante o atendimento, como depois, na questão de esterilização. [...] A gente tem todo o protocolo que é seguido aqui." (Profissional 14)

\section{Representações sociais do risco e estratégia preventiva na segurança do paciente na Estratégia Saúde da Família}

Nesta categoria, os profissionais salientaram os riscos existentes na Estratégia Saúde da Família, além de medidas para redução de riscos, podendo ser de forma individual ou coletiva. Assim, constatou-se que os riscos para os pacientes são bem relevantes nas suas práticas, podendo levá-los a uma exposição cruzada ou a ter outros riscos, o que evidenciam as falas a seguir:

"[...] na administração de medicamento [...] o paciente pode ter uma reação alérgica, uma má aplicação pode causar hematomas, pode causar danos." (Profissional 6)

“[...] risco de constrangimento, pode ter risco de exposição, exposição pública de algum tipo de problema que o paciente possa vir a ter [...]" (Profissional 9)

"[...] muitas vezes não tem papel pra cobrir a maca, a gente usa bata e fica a bata até o último paciente." (Profissional 17)

[...] a gente atende todo tipo de doença infecciosa, desde viroses até tuberculose, sarampo, meningite, catapora, tudo, e todo mundo espera junto... [...]" (Profissional 18)

Diante disso, destacam-se as estratégias preventivas na utilização adequada das técnicas, a realização da desinfecção e esterilização, além de orientações sobre o tema com os colegas para uma melhor conduta na segurança do paciente, conforme as falas:

"[...] a cadeira a gente enrola com insulfilm [...]" (Profissional 1)

"[...] desinfecção sempre, a cada paciente que sai a gente vai fazendo [...]" (Profissional 3)

"tentar fazer da melhor forma possivel utilizando a técnica. Orientar nossos colegas de trabalho, discutindo como fazer, o que a gente pode melhorar." (Profissional 6)

"[...] a questão da esterilização do material e a utilização de material descartável também [...]" (Profissional 9)

\section{Fragilidades da segurança do paciente na Estratégia Saúde da Família}

Nesta categoria são discutidos aspectos sobre as fragilidades que podem influenciar na prática do atendimento do profissional e as dificuldades na atuação de implementação sobre a segurança do paciente.

Assim, demostrou-se que a falta de materiais para o andamento do atendimento e a estrutura física influenciam, sendo pequenos fatores para que não deem continuidade ao atendimento correto:

"[...] às vezes, a gente tem uma falta de insumos; às vezes, a gente tem uma falta do próprio aparelho de 
pressão por conta da demanda ser grande." (Profissional 7)

"Acho muito ruim a falta da estrutura do posto; a gente, infelizmente, atende sem papel toalha, quando tem papel toalha a gente utiliza o mesmo [...]" (Profissional 18)

Diante disso, constatou-se que existem dificuldades, principalmente, em termos de acessibilidade aos equipamentos de proteção individual (EPI) e no uso deles, provocando alterações nas rotinas das consultas:

“[...]... em termos de EPI, essas coisas assim, tem muito a melhorar; a gente só consegue pedindo à coordenação, pedindo à farmácia, pedindo para solicitar. Não tem fácil em nenhum consultório, a gente acaba se expondo [...]" (Profissional 13)

"[...] para uma coleta de exame preventiva, não existe bata suficiente para todas [...]" (Profissional 17)

\section{DISCUSSÃO}

Uma das categorias encontradas no presente estudo, "Representações sociais da segurança do paciente na Estratégia Saúde da Família", aponta representações sociais objetivas e subjetivas do conhecimento, como os protocolos direcionados para a segurança do paciente, a rotina de trabalho na atenção primária à saúde e o acolhimento que lida com valores e significados do profissional e usuário e um compartilhamento de saberes, ancorados na formação e na legislação acerca da temática. A partir dos relatos, os profissionais demonstraram que possuem um entendimento essencial referente à temática, à importância na assistência e nas estratégias vivenciadas, e que dispõem de uma compreensão do significado de segurança do paciente na atenção primaria à saúde.

A principal estratégia para promover a segurança do paciente e suas implicações é que o profissional tenha conhecimento do seu significado e objetivos, para uma melhoria diária no seu desenvolvimento. A abordagem constante com treinamentos e capacitações sobre a cultura de segurança deve ser implementada na Atenção Primaria à Saúde, como forma de preparar o profissional para o atendimento, já que alguns têm a incerteza do que é, os efeitos adversos e seu impacto para o cliente ${ }^{(24)}$.

Assim, os profissionais de saúde devem seguir as normatizações recomendadas pela Organização Mundial da Saúde (OMS) e pelo Ministério da Saúde (MS), no intuito de garantir a segurança do paciente. Dessa forma, todos os protocolos e medidas de segurança devem ser seguidas criteriosamente, de acordo com que é exigido pelo núcleo de segurança do paciente ${ }^{(25)}$.

Além das orientações, os cuidados prestados antes, durante e depois se mostram relevantes para o alcance e a manutenção da segurança dos pacientes. Essas ações devem fazer parte da rotina de trabalho dos profissionais de saúde, independente do serviço de saúde de que faça parte e da categoria profissional, pois precisam garantir sua segurança de saúde e do paciente. O conhecimento das normas e rotinas é um fator primordial para que as ações voltadas para a segurança do paciente aconteçam na prática. Outros autores ${ }^{(26)}$ identificaram em sua pesquisa uma divergência na percepção da cultura de segurança pelos profissionais de saúde da atenção primária, denotando a importância do planejamento conjunto de estratégias de assistência à saúde.

Corroborando a atual pesquisa, foi identificado em outro estudo que a segurança do paciente está diretamente ligada ao ambiente, ações, infecção e protocolo, revelando a importância do conhecimento no aspecto da prática profissional e do cotidiano no trabalho no serviço de saúde ${ }^{(27)}$.

$\mathrm{Na}$ categoria "Representações sociais do risco e estratégia preventiva na segurança do paciente na Estratégia Saúde da Família", as representações sociais sinalizaram os riscos a que o paciente estão submetidos, como administração de medicamentos, constrangimentos e rompimento de medidas de biossegurança (distanciamento, esterilização e desinfecção), além da importância do desenvolvimento de estratégias para a minimização desses riscos. Esse achado corrobora os achados de um estudo ${ }^{(28)}$ acerca das representações sociais da equipe de enfermagem sobre biossegurança na Atenção Primária, permitindo levantar que o conhecimento elaborado e compartilhado pelos participantes é enraizado no decorrer de sua prática profissional, estando ancorado no decurso do cotidiano, no qual esses profissionais atrelaram o conceito de biossegurança à exposição e a acidentes na realização de suas atividades, devido às dificuldades encontradas no cotidiano do trabalho.

Os profissionais investigados no presente estudo perceberem a importância de implementar a segurança do paciente no serviço de saúde, garantindo uma assistência contínua e de qualidade. Os usuários também identificam essa importância, conforme atesta uma pesquisa ${ }^{(29)}$ realizada na Suécia, que destacou as percepções dos pacientes sobre o risco de dano na atenção primária à saúde, possuindo como fatores relevantes: a continuidade do atendimento, pois quando o cuidado torna-se fragmentado compreendem que aumentam o risco para a saúde do paciente; as 
rotinas de acompanhamento, devido aos pacientes se sentirem responsáveis para que o acompanhamento aconteça; o envolvimento do paciente, pois quando é baixo, dificulta o acesso ao serviço e diagnóstico precoce; a presença de erros, como na dosagem de medicamentos; o atendimento não qualificado, ou seja, o profissional demonstrou dúvidas, não realizando anamnese e exame físico detalhado.

Uma pesquisa realizada no Reino Unido destacando o monitoramento da segurança do paciente na atenção primária identificou os seguintes fatores como essenciais para garantir a segurança do paciente: o aumento da equipe e do recurso; realizar auditorias e compartilhar os resultados; compartilhar registros eletrônicos; autoavaliação e avaliação entre pares; sistema de relatório de eventos adversos; evitar danos e eventos adversos ${ }^{(30)}$. Essas medidas são essenciais e importantes para minimizar os riscos durante o atendimento, por isso é necessário que os profissionais conheçam a implementem em suas práticas, independentemente do grau de complexidade do serviço, garantindo, assim, a segurança do paciente e uma assistência de saúde adequada e qualificada.

O desenvolvimento do cuidado seguro relacionado à ética profissional favorece a redução de danos/riscos ao paciente, pois seu compromisso com sua assistência (adequada) e a continuidade do cuidado evita a exposição do paciente. A empatia e a disposição do trabalhador, bem como sua ética a respeito da situação, são maneiras de se estabelecer vínculos a fim de promover a continuidade do cuidado. A postura e a maneira de escutar e de encarar o inesperado se constituem em afirmação de relações entre funcionário e usuário, principalmente quando as pessoas buscam assistência na ausência de agendamentos ou sem consultas ${ }^{(31)}$.

Na categoria temática "Fragilidades da segurança do paciente na Estratégia Saúde da Família", os entrevistados da atual pesquisa expressam as representações sociais centradas na falta de material e no ambiente (estrutura física), implicando diretamente na assistência individual e contínua ao paciente.

Os participantes relataram a falta de insumos e de estrutura do ambiente que interferem na prática, colaborando para o aumento de risco ao paciente. Outros pesquisadores ${ }^{(32)}$ estão de acordo com essa perspectiva, pois muito pouco ou quase nada adianta o paciente ser cuidado por uma equipe interdisciplinar, que visa à promoção da sua saúde e do seu bem-estar, se a estrutura não colabora com o funcionamento adequado para a prestação de uma assistência de qualidade e segura.

No presente estudo, conforme a fala dos participantes, as iniciativas voltadas à segurança do paciente na APS ainda são incipientes e limitadas, e os profissionais percebem, na maioria dos domínios, a cultura como negativa. Apesar de possuir documentos internacionais e nacionais que discutem a temática, sua implementação na prática ainda está distante, sendo importante ser abordada desde a formação do profissional, destacando sua relevância para o paciente, profissional de saúde e o serviço ${ }^{(3)}$. É vital compreender a magnitude e a natureza nos cuidados primários, pois uma proporção significativa dos cuidados de saúde é oferecida nesse cenário, mas há pouca clareza sobre as maneiras mais eficazes de abordar questões de segurança nesse nível. Portanto, melhorar a segurança na atenção primária é essencial quando se busca alcançar a cobertura universal de saúde e a sustentabilidade dos cuidados de saúde. O governo deve garantir que os cuidados prestados neste ambiente sejam seguros, eficazes e focados nas necessidades do paciente, pois o atendimento primário seguro melhora a saúde e o bem-estar de indivíduos, comunidades e sociedades ${ }^{(4)}$.

As limitações do estudo dizem respeito à amostra reduzida de profissionais (decorrente da recusa em querer participar do estudo), mas estimulam reflexões e abrem perspectivas para novas pesquisas.

\section{CONSIDERAÇÕES FINAIS}

O estudo permitiu apreender as representações sociais da segurança do paciente sob a ótica dos profissionais de saúde, que despontaram que ancoram suas compreensões de segurança do paciente na organização e dinâmica do seu processo de trabalho, mediante subsídios e estratégias.

As representações sociais evidenciam que, no cotidiano de trabalho, as ações em prol da segurança do paciente são conduzidas por evidência científica; por outro lado, surgem dimensões subjetivas, pois os profissionais parecem ter incorporado uma cultura de segurança positiva, adotando o compromisso por um cuidado de qualidade, em detrimento do agir espontaneamente.

\section{CONFLITOS DE INTERESSE}

Os autores declaram a inexistência de conflitos de interesses, tanto na execução das ações do projeto de pesquisa como na escrita do manuscrito. 


\section{CONTRIBUIÇÕES}

Emanuel Alves do Nascimento contribuiu com a elaboração e delineamento do estudo; a aquisição, análise e interpretação dos dados; e a redação e/ou revisão do manuscrito. Charlyanne Diógenes Brito e Daiany Dântara de Sousa Barbosa contribuíram com a aquisição dos dados. Samira Valentim Gama Lira de Alencar contribuiu com a análise e interpretação dos dados; e a redação e/ou revisão do manuscrito. Lívia de Andrade Marques, Geisy Lanne Muniz Luna, Débora Rodrigues Guerra Probo e Ricardo Augusto da Silva Probo contribuíram com a redação e/ou revisão do manuscrito.

\section{REFERÊNCIAS}

1. Ministério da Saúde (BR), Agência Nacional de Vigilância Sanitária. Resolução de Diretoria Colegiada - RDC $n^{\circ} 36$, de 25 de julho de 2013. Institui ações para a segurança do paciente em serviços de saúde e dá outras providências [Internet]. Brasília: Ministério da Saúde; 2013 [acesso em 2020 Mar 14]. Disponível em: http:// bvsms.saude.gov.br/bvs/saudelegis/anvisa/2013/rdc0036_25_07_2013.html

2. World Health Organization. Patient safety: patient safety solutions preamble [Internet]. 2007 [acesso em 2020 Mar 2]. Disponível em: https://www.who.int/patientsafety/events/07/02_05_2007/en/

3. Fausto MCR, Rizzoto MLF, Giovanella L, Seidl H, Bousquat A, Almeida PF, et al. O futuro da atenção primária à saúde no Brasil. Saúde Debate. 2018;42(especial 1):12-7.

4. Ministério da Saúde (BR). Política Nacional de Promoção da Saúde: PNPS: Anexo I da Portaria de Consolidação no 2, de 28 de setembro de 2017, que consolida as normas sobre as políticas nacionais de saúde do SUS. Brasília: Ministério da Saúde; 2018.

5. Kuriakose R, Aggarwal A, Sohi RK, Goel R, Rashmi NC, Gambhir RS. Patient safety in primary and outpatient health care. J Family Med Prim Care. 2020;9(1).

6. World Health Organization. Patient safety: global action on patient safety [Internet]. 2019 [acesso em 2020 Jun 24]. Disponível em: https://apps.who.int/gb/ebwha/pdf_files/WHA72/A72_26-en.pdf6

7. World Health Organization. World alliance for patient safety: forward programme 2005 [Internet]. Genebra: WHO; 2004 [acesso em 2020 Jun 24]. Disponível em: https://apps.who.int/iris/handle/10665/43072

8. Marchon SG, Mendes WV Júnior. Segurança do Paciente na Atenção Primária à Saúde [Slides] [Internet]. Rio de Janeiro: Proqualis; 2016 [acesso em 2020 Jun 8]. Disponível em: https://proqualis.net/aula/ seguran\%C3\%A7a-do-paciente-na-aten\%C3\%A7\%C3\%A3o-prim\%C3\%A1ria-\%C3\%A0-sa\%C3\%BAde

9. Nora $C R D$, Beghetto $M G$. Desafios da segurança do paciente na atenção primária à saúde: revisão de escopo. Rev Bras Enferm. 2020;73(5).

10. Castro GLT, Tourinho FSV, Martins MFSV, Medeiros KS, Ilha P, Santos VEP. Proposta de passos para a segurança do paciente no atendimento pré-hospitalar móvel. Texto \& Contexto Enferm. 2018;27(3).

11. Ministério da Saúde (BR). Segurança do Paciente no domicílio. Brasília: Ministério da Saúde; 2016.

12. Macedo SMK, Barboza ARCA, Borges F, Figueiredo KC, Peres AM, Assis F. Patient safety culture: evaluation of nurses in primary health care. Enferm Glob. 2019 oct;(56):387-97.

13. Vincent $C$, Amalberti R. Cuidado de Saúde mais Seguro: estratégias para o cotidiano do cuidado. Rio de Janeiro: Proqualis; 2016.

14. Silva APF, Backes DS, Magnagio TSBS, Colome JS. Segurança do paciente na atenção primária: concepções de enfermeiras da estratégia de saúde da família. Rev Gaúcha Enferm. 2019 jan;40(spe).

15. Rodrigues AS, Oliveira JF, Suto CSS, Coutinho MPL, Paiva MS, Souza SS. Cuidado à mulher envolvida com drogas: representações sociais de enfermeiros. Rev Bras Enferm. 2017;70(1):65-72.

16. Marchon SG, Mendes WV Júnior, Pavão ALB. Characteristics of adverse events in primary health care in Brazil. Cad Saúde Pública [Internet]. 2015 [acesso em 2018 Mar 22];31(11):2313-30. Disponível em: https:// www.scielo.br/scielo.php?script=sci_arttext\&pid=S0102-311X2015001102313\&lng=pt\&tlng=pt17

17. Minayo MCS. O desafio do conhecimento: pesquisa qualitativa em saúde. $14^{\mathrm{a}}$ ed. Rio de Janeiro: Hucitec; 2015. 
18. Moscovici S. A representação social da psicanálise. Rio de Janeiro: Zahar; 1978.

19. Moscovici S. On social representation. In: Forgas J. Social Cognition. London: Academic Press; 1981. p. 181209.

20. Jodelet D. Representações sociais: um domínio em expansão. In: Jodelet D, organizador. As Representações Sociais. Rio de Janeiro: EdUERJ; 2001. p. 17-44.

21. Prefeitura de Municipal Fortaleza. Postos de Saúde [Internet]. [data desconhecida] [acesso em 2020 Jan 4]. Disponível em: https://saude.fortaleza.ce.gov.br/postos-de-saude

22. Bardin L. Análise de Conteúdo. São Paulo: Edições 70; 2016.

23. Conselho Nacional de Saúde (BR). Resolução n. 466, de 12 de dezembro de 2012 [Internet]. Brasília: CNS; 2012 [acesso em 2020 Jun 11]. Disponível em: http://conselho.saude.gov.br/resolucoes/2012/Reso466.pdf

24. Mesquita KO, Silva LCC, Lira RCM, Freitas CASL, Lira GV. Segurança do Paciente na Atenção Básica: revisão integrativa. Cogitare Enferm. 2016;21(2):1-8.

25. Chimenez PA, Bastos TFC. Segurança do paciente: percepção dos acadêmicos de enfermagem [trabalho de conclusão de curso]. Fortaleza: Universidade de Fortaleza; 2016.

26. Macedo LL, Silva AMR, Silva JFM, Haddad MCFL, Girotto E. A cultura em torno da segurança do paciente na atenção primária à saúde: distinções entre categorias profissionais. Trab Educ Saúde. 2019;18(1).

27. Barreto RS, Servo MLS, Ribeiro AMVB. Representações sociais sobre segurança do paciente na ótica de enfermeiras intensivistas. Rev Bahiana Enferm. 2020;34.

28. Sousa AF, Queiroz AA, Oliveira LB, Moura ME, Batista OM, Andrade D. Representações sociais da enfermagem sobre biossegurança: saúde ocupacional e cuidar cuidar prevencionista. Rev Bras Enferm. 2016;69(5):864-71.

29. Fernholm R, Holzmann MJ, Malm-Willadsen K, Pukk Härenstam K, Carlsson AC, Nilsson GH, et al. Patient and provider perspectives on reducing risk of harm in primary health care: a qualitative questionnaire study in Sweden. Scand J Prim Health Carev. 2020;38(1):66-74.

30. Samra R, Car J, Majeed A, Vincent C, Aylin P. How to monitor patient safety in primary care? Healthcare professionals' views. JRSM Open. 2016;7(8):1-8.

31. Agencia Nacional de Vigilância Sanitária (BR). Assistência segura: uma reflexão teórica aplicada à prática. Brasília: Anvisa; 2017.

32. Costa DVS, Fragoso LVC, Queiroz PA, Carvalho SMA, Costa DVS, Freitas MMC. Nursing Contributions to the Patient's Safery in The Intensive Care Unit: an intregrave review. J Nurs UFPE. 2016;10(6):2177-88.

33. Schmidt CR, Santos FP, Loro MM, Souza MM, Kolankiewicz ACB. Atenção Primária a Saúde como cenário de avaliação da cultura de segurança do paciente. Rev Pre Infec Saúde. 2019;5.

\section{Endereço para correspondência:}

Samira Valentim Gama Lira de Alencar

Universidade de Fortaleza - Centro de Ciências da Saúde

Av. Washington Soares, 1321

Bairro: Edson Queiroz

CEP: 60811-905 - Fortaleza - CE - Brasil

E-mail:vagali@uol.com.br

Como citar: Alencar SVGL, Nascimento EA, Brito CD, Barbosa DDS, Marques LA, Luna GLM, et al. Segurança do paciente: representações sociais na visão dos profissionais da atenção primária à saúde. Rev Bras Promoç Saúde. 2021;34:11636. 